\title{
Onlinebasierte, qualifizierte Zweitmeinung für Patienten mit kolorektalem Karzinom - eine Pilotstudie der Felix Burda Stiftung in Zusammenarbeit mit dem Netzwerk gegen Darmkrebs Online-based, qualified second opinion for patients with colorectal cancer - A pilot study of the Felix Burda Foundation in co-operation with the Network against Colorectal Cancer
}

\section{Autoren}

Ursula Berger ${ }^{1}$, Udo E. Beckenbauer², Caroline Rimkus³ , Franz G. Bader ${ }^{4}$, Thomas Theodor Werner Seufferlein ${ }^{5}$, Christa Maar ${ }^{6}$

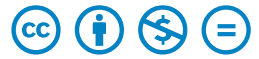

Institute

1 Institut für medizinische Informationsverarbeitung, Biometrie und Epidemiologie, Ludwig-MaximiliansUniversität München

2 HMO Health Management Online AG

3 Abteilung für Allgemein-, Viszeral- und Kinderchirurgie, Universitätsmedizin Göttingen

4 Klinik für Allgemein-, Viszeral- und Minimalinvasive Chirurgie, Isarklinikum München

5 Klinik für Innere Medizin I, Universitätsklinikum Ulm

6 Hubert Burda Stiftung/Felix Burda Stiftung

Schlüsselwörter

qualifizierte Zweitmeinung, kolorektales Karzinom,

Elektronische Patientenakte, Lebensqualität, interdisziplinäres Tumorboard

Key words

qualified second opinion, colorectal carcinoma, electronic patient record, quality of life, tumour board

eingereicht 23.01.2020

akzeptiert 08.04.2020

Bibliografie

DOI https://doi.org/10.1055/a-1157-9787

Online-Publikation: 25.5.2020

Z Gastroenterol 2020; 58: 556-563

(c) Georg Thieme Verlag KG, Stuttgart · New York ISSN 0044-2771

Korrespondenzadresse

Dr. Ursula Berger,

Institut für medizinische Informationsverarbeitung, Biometrie und Epidemiologie, Ludwig-Maximilians-Universität

München, Marchioninistr. 15, 81377 München

Tel.: +49/89/440077486

Ursula.Berger@LMU.de $\circledast$

Supplemental Material:

https://doi.org/10.1055/a-1157-9787

\section{ZUSAMMENFASSUNG}

Hintergrund Moderne, individualisierte Therapien können das Überleben von Patienten mit kolorektalem Karzinom verbessern. Jedoch werden nicht alle Patienten zur Behandlung in ein zertifiziertes Darmkrebszentrum überwiesen, wo ihre Therapie, unterstützt durch ein Tumorboard, leitliniengerecht durchgeführt wird. Daher wird derzeit die Zweitmeinung bei Krebserkrankungen diskutiert. Diese Studie beschreibt Ergebnisse einer Pilotstudie hinsichtlich der Machbarkeit und des Bedarfs einer strukturierten, onlinebasierten, qualifizierten Zweitmeinung für Patienten mit kolorektalem Karzinom.

Methoden Im Rahmen einer 15-monatigen Pilotphase zwischen 2009 und 2011 konnten Patienten mit kolorektalem Karzinom mittels einer onlinebasierten Elektronischen Patientenakte, unterstützt durch einen Case Manager, kostenfrei eine qualifizierte Zweitmeinung eines Tumorboards einholen. Ihre Zufriedenheit und ihre Lebensqualität (EORCT QLQ-C30) wurden über ein Jahr nachbeobachtet.

Ergebnisse In 95\% der Fälle konnten eine vollständige Elektronische Patientenakte und eine Zweitmeinung erstellt werden. Weniger als die Hälfte der Teilnehmer erhielt die erste Therapieempfehlung aus einer Klinik mit Tumorboard. Die Erstmeinung konnte in $40 \%$ der Fälle durch die Zweitmeinung bestätigt werden, in $33 \%$ zeigte sich ein teilweises, in $27 \%$ ein deutliches Abweichen. Bei Umsetzung der abweichenden Zweitmeinung verbesserte sich die Lebensqualität der Patienten.

Schlussfolgerung Die Erstellung einer onlinebasierten, qualifizierten Zweitmeinung durch ein interdisziplinäres Tumorboard ist technisch und logistisch gut durchführbar. Die onlinebasierte Zweitmeinung könnte zukünftig die Qualität der Behandlung von Patienten mit kolorektalem Karzinom deutlich verbessern und damit ihre Lebensqualität erhöhen. 


\section{ABSTRACT}

Background Modern, individualised therapies can improve the survival of patients with colorectal cancer. However, not all patients are referred for treatment to a certified colorectal cancer centre, where a tumor board supports the implementation of their therapy in accordance to guidelines. This study examines the feasibility and demand of a structured, online-based, qualified second opinion for patients with colorectal cancer.

Method A 15-month pilot study between 2009 and 2011, offered patients with colorectal cancer to obtain a qualified second opinion of a tumour board based on an electronic patient record completed online with the assistance of a case manager. Life-satisfaction levels and quality of life (EORCT QLQ-C30) of the participants has been monitored for a year.
Results In $95 \%$ of the cases, a complete electronic patient record and a second opinion could be generated. Less than half of the participants received their first therapy recommendation from a clinic with a tumour board. The second opinion confirmed the initial medical opinion in $40 \%$ of the cases $33 \%$ showed a partial and $27 \%$ showed a significant deviation. In case of a deviation, the implementation of the second opinion improved the patients' quality of life.

Conclusion Generating an online-based, qualified second opinion by an interdisciplinary tumour board is technically and logistically well feasible. The online-based second opinion could significantly improve the quality of treatment for patients with colorectal cancer in the future and thus improve their quality of life.

\section{Einleitung}

Jährlich erkranken in Deutschland 62400 Menschen an einem kolorektalen Karzinom (KRK), 25700 sterben daran. Das KRK stellt bei Männern nach Lungenkrebs die zweithäufigste, bei Frauen nach Brust- und Lungenkrebs die dritthäufigste Ursache krebsbedingter Todesfälle dar [1]. Durch zunehmende Implementierung multimodaler Therapieverfahren und Einsatz medikamentöser Therapien, die eine individuelle und zielgerichtete Therapie erlauben, ist bei manchen Patienten eine Heilung auch in fortgeschrittenen Stadien möglich. Dies erfordert den Einsatz differenzierter, hochspezialisierter Diagnostik, eingebunden in ein interdisziplinäres Team von Ärzten. Dieser Austausch von Experten erfolgt in Zentren in der Regel in einem interdisziplinären Tumorboard. Die Deutsche Krebsgesellschaft hat in der Vergangenheit zur Etablierung solcher interdisziplinärer Tumorboards erheblich beigetragen. Bezüglich des KRK gibt es in Deutschland derzeit 279 zertifizierte Darmkrebszentren (Stand Juni 2019), die hohen Qualitätsanforderungen hinsichtlich Diagnostik und Therapie von KRK unterliegen. Die Voraussetzung zu einer Therapieentscheidung ist dort stets die interdisziplinäre Fallbesprechung in einem Tumorboard. In der täglichen Praxis werden aber längst nicht alle Patienten mit einem neu diagnostizierten KRK in einem zertifizierten Darmzentrum bzw. in einem interdisziplinären Tumorboard vorgestellt. Für das Gesamtüberleben und auch die Lebensqualität der Patienten mit einer malignen Erkrankung kann dies jedoch von entscheidender Bedeutung sein [2-5].

Zur Verbesserung der Versorgung von Patienten mit einem KRK wurde die Pilotstudie „Onlinebasierte, qualifizierte Zweitmeinung für Patienten mit kolorektalem Karzinom“ initiiert. Im Rahmen einer 15-monatigen Pilotphase konnten sich Patienten mit KRK mittels einer onlinebasierten Elektronischen Patientenakte (EPA) kostenfrei eine qualifizierte ärztliche Zweitmeinung in einem interdisziplinären Tumorboard einer an der Studie beteiligten Klinik einholen. Interessierte Patienten gelangten auf der Website www.darmkrebs.de über ein Anmeldeformular zu einem Case Manager (CM), der sie beim Zusammentragen der für die Zweitbegutachter notwendigen Unterlagen unterstützte und eine EPA erstellte, die dann in einer Tumorkonferenz diskutiert wurde. Die ausgesprochene Therapie- empfehlung wurde durch den CM dem Patienten und ggf. seinem behandelnden Arzt übermittelt.

Bisher gibt es wenige Studien zu Ergebnis und Nutzen von ärztlichen Zweitmeinungen [6]. Erste allgemeine Ergebnisse weisen jedoch auf einen Nutzen der Zweitmeinung hin [7]. Die vorliegende Pilotstudie sollte untersuchen, (a) inwieweit eine onlinebasierte Zweitmeinung machbar und plausibel ist bzw. von Betroffenen angenommen wird. Darüber hinaus sollte geklärt werden, welche Patienten Interesse an einer Zweitmeinung haben, (b) wie hoch die Übereinstimmung der Erstmeinung und der Zweitmeinung ist und welche Faktoren dies beeinflussen, (c) welche Entscheidungen die Patienten aufgrund der Zweitmeinung fällen und (d) wie sich diese auf ihre Lebensqualität und Zufriedenheit auswirkt.

\section{Methodik}

Im Rahmen des deutschlandweiten Pilotprojekts Zweitmeinungsstudie bot die Felix Burda Stiftung zwischen dem 7. Dezember 2009 und dem 3. März 2011 Patienten mit KRK auf der Patientenplattform www.darmkrebs.de die Möglichkeit an, sich kostenfrei eine qualifizierte Zweitmeinung des Tumorboards aus einer der zuvor deutschlandweit ausgewählten Gruppe von 30 spezialisierten Kliniken einzuholen (Anhang A1). Mittels eines Onlinefragebogens wurden von den Patienten zunächst Diagnose und Erkrankungsstadium angegeben und extern auf Plausibilität kontrolliert. Einschlusskriterien zur Studie waren Erstdiagnosen von Rektumkarzinomen unabhängig von ihrem Stadium, Erstdiagnosen von metastasierten Kolonkarzinomen, Lokal- und Metastasenrezidive, Zweittumoren sowie Kolontumoren in Behandlung. Der Patient wurde telefonisch von einem Case Manager (CM) kontaktiert und über die Studie aufgeklärt. Nach Einholung einer schriftlichen Einwilligungserklärung führte der CM das erste telefonische Interview (Baseline) durch und betreute die anschließende Zusammenstellung der für eine Zweitmeinung erforderlichen Daten und Dokumente des Patienten in einer strukturierten, onlinebasierten Elektronischen Patientenakte (EPA). Der Patient und mit dessen Einverständnis auch seine Ärzte erhielten Zugang zur EPA und konnten Daten und Dokumente entweder selbst direkt online ein- 
tragen oder sie per Post oder Fax an den CM übermitteln, der sie dann (ggf. nach Aufbereitung) in die EPA eintrug. Diese onlinebasierten Daten wurden dann als EPA an eines der teilnehmenden Tumorboards zur Einholung einer qualifizierten Zweitmeinung übermittelt und dort auf Plausibilität überprüft. Bilddateien wurden den teilnehmenden Kliniken per CD zur Verfügung gestellt. Das interdisziplinäre Tumorboard gab eine schriftliche Behandlungsempfehlung entsprechend der aktuellen S3-Leitlinie [8] ab, die dann wiederum in die EPA des Patienten eingetragen wurde. Der CM übermittelte die Therapieempfehlung an den Patienten und mit dessen Einverständnis auch an den Primärarzt. Zudem konnte auf Patientenwunsch eine Vermittlung zum verantwortlichen Oberarzt der Klinik, die die Zweitmeinung erstellt hatte, erfolgen. Nach drei, sechs und zwölf Monaten wurden die Patienten durch standardisierte Telefoninterviews nachbeobachtet. Die EPA musste mindestens die folgenden Informationen enthalten: Befunde (Erstdiagnose und Lokalisation des Primärtumors, TNM-Klassifikation nach UICC [9], Koloskopie, Computertomografie), Laborwerte und ggf. Tumormarker, Anamnese, Begleiterkrankungen, bisherige Therapie, Medikation.

\section{Variablen}

Für alle Patienten wurden Daten zu Alter und Geschlecht, zu Tumorerkrankungen bei Verwandten 1. oder 2. Grades, zum bisherigen Krankheitsverlauf und zum aktuellen Gesundheitszustand (u. a. Schmerzen), zu bisherigen Therapien (Operationen, Chemound Strahlentherapien), zur Art der Erstmeinungs- und der Zweitmeinungsklinik sowie zur Größe des Wohnorts und zum höchsten Bildungsabschluss des Patienten erhoben. Die Lebensqualität und der subjektive Gesundheitszustand wurden bei der Erstbefragung und nach 3, 6 und 12 Monaten mit dem EORTC Quality of Life Questionnaire-Core-30 (QLQ-C30) der European Organization for Research and Treatment of Cancer (EORTC QLQ-C30 Version 3.0) $[10,11]$ auf einer 7-Punkte Skala gemessen. Der sich daraus ergebende Score ist auf Werte zwischen 0 und 100 standardisiert, wobei ein höherer Wert auf eine höhere Gesundheit bzw. Lebensqualität hinweist. Im Follow-up von 3 Monaten wurden die Patienten zudem nach ihrer Entscheidung für eine weitere Behandlung befragt. Verbunden damit war die Frage nach der Zufriedenheit mit dieser Entscheidung (,ja“, „nein“). Das zweitmeinungsgebende Tumorboard bewertete zudem die Übereinstimmung zwischen Erst- und Zweitmeinung in einem Onlineformular.

\section{Statistische Methoden}

Die Vergleiche der Charakteristika von Patienten mit Kolonkarzinom (ICD-10-GM C18) und Patienten mit einem Rektumkarzinom (ICD10-GM C19 oder (20) und von Patienten ohne und mit Abweichung zwischen Erst- und Zweitmeinung basieren auf dem Exakten Test nach Fisher. Veränderungen der Lebensqualität (EORTC QLQ-C30) zwischen Erstbefragung und dem 3-Monats-Follow-up wurden mittels t-Tests geprüft und in Error-Plots dargestellt und die Effekte unterschiedlicher Charakteristika in einer multiplen Regressionsanalyse untersucht. Die Veränderung des Gesundheitszustands insgesamt über alle vier Erhebungszeitpunkte wurde in gemischten Regressionsmodellen analysiert. Für alle Tests wurde ein Testergebnis mit einem p-Wert <0,05 als signifikant interpretiert. Alle Analysen wurden in SPSS (Version 17) oder in R (Version 3.1.1) durchgeführt.

\section{Ergebnisse}

Bei 112 der 156 Anfragen über die Onlineplattform, die die Einschlusskriterien erfüllten, kam ein Erstkontakt zustande. 5 Patienten verstarben tumorbedingt vor Einholung der Zweitmeinung und 7 Patienten entschieden sich gegen eine Zweitmeinung. Bei 5 der 100 verbleibenden Patienten konnten die Befunde nicht vollständig zusammengestellt werden. Die verbliebenen $95 \mathrm{~Pa}$ tienten erhielten alle eine onlinebasierte Zweitmeinung ( Abb. 1). Der Hälfte der Patienten konnte innerhalb von höchstens 16 Tage nach dem Baseline-Interview die Zweitmeinung übermittelt werden (mediane Bearbeitungszeit). Bei $73 \%$ lag eine fortgeschrittene Tumorerkrankung mit Metastasen vor (UICC Stadium IV), und die Mehrheit hatte eine Operation, Chemotherapie und/oder Strahlentherapie hinter sich. Etwa ein Drittel aller Patienten gab Schmerzen an. Einen Gymnasial- oder Universitätsabschluss hatten $38 \%, 16 \%$ keinen oder einen Hauptschulabschluss. Über $70 \%$ der Teilnehmer lebten in einem Ort mit weniger als 100.000 Einwohnern. (Weitere Charakteristika sind in - Tab. 1 dargestellt.)

\section{Abweichung von Erstmeinung und Zweitmeinung}

Ihren primären Therapievorschlag (Erstmeinung) haben knapp $56 \%$ der Teilnehmer $(n=53)$ von einer Klinik ohne Tumorboard oder Praxis erhalten, $34 \%$ ( $n=33$ ) von einem zertifizierten Darmkrebszentrum oder einer Großstadtklinik und ca. $10 \%(n=9)$ von einer Universitätsklinik. Insgesamt wurde die Erstmeinung in $40 \%$ $(n=38)$ durch die Zweitmeinungsanfragen bestätigt, in $33 \%$ $(n=31)$ bestand ein „teilweises“ Abweichen, in $27 \%(n=26)$ ein "deutliches“ Abweichen ( $>$ Tab. 1).

Deutliche Abweichungen zwischen Erst- und Zweitmeinung gab es häufiger bei Erstmeinungen aus Praxen oder Kreiskliniken (36\% von 53) im Vergleich zu Erstmeinungen aus Universitätskliniken, Darmzentren oder Großstadtkliniken (17\% von 42). Geringfügigere Abweichungen betrafen eher Erstmeinungen aus Universitätskliniken, Darmzentren oder Großstadtkliniken (41\% von 42 vs. $27 \%$ von 53). Am häufigsten betrafen die Modifikationen die Zusammensetzung der Chemotherapie (48\%) und eine Therapieänderung zugunsten eines operativen Vorgehens (39\%). Bei Letzterem wurde durch die Tumorboards häufig eine Resektion in kurativer Absicht vorgeschlagen, während die Situation vom Erstbehandler bereits als nicht mehr chirurgisch therapierbar eingestuft wurde. In $3 \%$ betrafen die Modifikationen die Bestrahlungstherapien, und bei weiteren $9 \%$ kam es zu sonstigen Abweichungen (zum Beispiel Best-supportive-Care-Maßnahmen). Es gab keine wesenlichen Unterschiede zwischen kurativen (UICC I-III) und palliativen (UICC IV) Fällen bezüglich der Häufigkeit der Abweichungen (Anhang A2).

\section{Follow-up}

Innerhalb der ersten 3 Monate verstarben 15 der 95 Patienten; 23 Patienten gaben trotz mehrfacher Nachfrage keine weitere Rückmeldung ( $\triangleright$ Abb. 1). Von den 57 Patienten mit einer Rückmeldung wich bei $61 \%(n=35)$ die Zweitmeinung ganz oder teilweise von der ersten Therapieempfehlung ab. Die Hälfe von ihnen ( $n=17$ ) gab nach drei Monaten an, die Therapie gewechselt zu ha- 


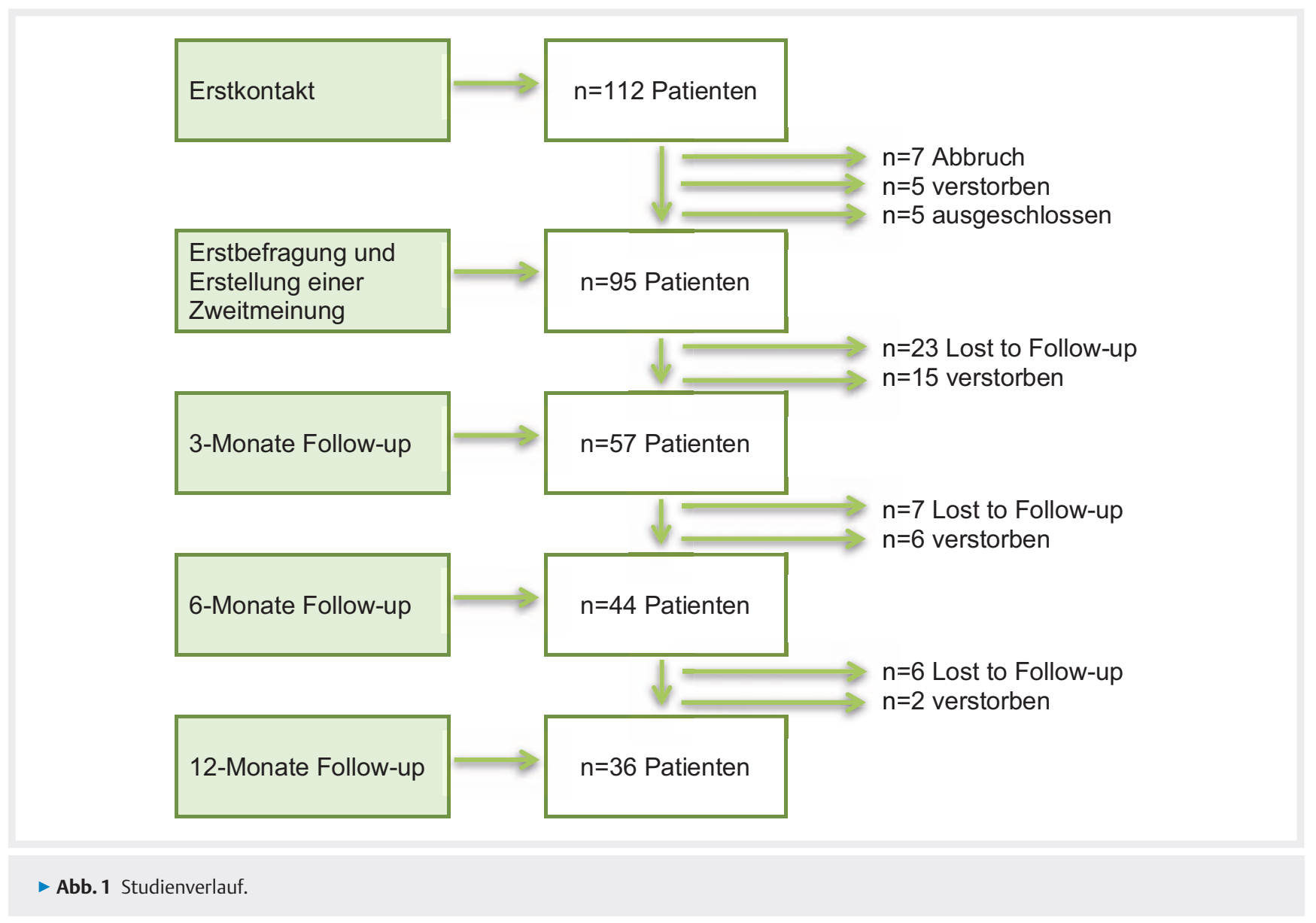

ben. Im Gegensatz dazu gaben bei Bestätigung der Ersttherapie durch die Zweitmeinung ( $\mathrm{n}=22,39 \%$ ) nur drei Patienten (14\%) an, ihre Therapie gewechselt zu haben. $91 \%(n=52)$ der Patienten des 3-Monats-Follow-up waren mit der von ihnen getroffenen Therapieentscheidung zufrieden ( $\vee$ Tab. 2). Für 46 Patienten lagen Angaben zur Veränderung des allgemeinen Gesundheitszustands nach drei Monaten vor. Bei Patienten, deren Zweitmeinung von der Erstmeinung grundlegend oder teilweise abwich, hatte sich der allgemeine Gesundheitszustand nach drei Monaten signifikant verbessert ( $\triangleright$ Abb. 2). Dabei spielt das UICC-Stadium keine Rolle (Anhang A2). Die Veränderung des Gesundheitszustands ist außerdem mit dem Gesundheitszustand bei der Erstbefragung assoziiert ( $\triangleright$ Tab. 3): Patienten, die bei Eintritt in die Studie einen höheren Gesundheitsstatus angaben, erreichten weniger Verbesserung als Patienten mit anfangs niedrigerem Gesundheitszustand. Darüber hinaus profitierten vor allem Frauen und Patienten $<60$ Jahre. Werden alle Follow-up-Zeitpunkte gemeinsam betrachtet, zeigte sich zudem, dass sich der Gesundheitszustand auch insgesamt über die 3, 6 und 12 Monate verbesserte.

\section{Diskussion}

Das Recht von Patienten auf eine ärztliche Zweitmeinung wurde in Deutschland Mitte 2015 mit dem Versorgungsstärkungsgesetz (§27b SGB V) gesetzlich verankert. Die vorliegende, zwischen 2009-2011 durchgeführte Pilotstudie zur qualifizierten Zweit- meinung für Patienten mit einem kolorektalen Karzinom untersucht erstmals den Bedarf, die Durchführbarkeit und die Effekte eines onlinebasierten Zweitmeinungsangebots in Deutschland. Das Zweitmeinungsangebot stieß damit bereits vor der gesetzlichen Verankerung auf großes Interesse bei Krebspatienten aus allen Regionen, Alters- und Bildungsschichten, obwohl sie nicht flächendeckend beworben worden war. Den Zugang zur Zweitmeinung fanden die Patienten über die Internetseite www.darm krebs.de. Das Medium Internet hat in den vergangenen Jahrzehnten als Informationsquelle in Gesundheitsfragen deutlich mehr Nutzer gewonnen [12], jedoch fällt es Patienten schwer, vertrauenswürdige Informationen auszuwählen [13]. Empathie hat einen großen Einfluss auf das Vertrauen der Patienten und damit auf ihre Zufriedenheit bei der Onlinesuche nach Gesundheitsinformationen [14]. Eine zentrale Rolle auf dem Weg zur qualifizierten Zweitmeinung spielte - auch aus diesem Grund - der Case Manager. Seine Aufgabe bestand in der individuellen Betreuung, der Unterstützung bei der Vervollständigung der strukturierten EPA und der Übermittlung der Therapieempfehlungen des externen Tumorboards. Zudem half er bei der Vermittlung von Experten, kommunizierte mit den behandelnden Ärzten des Patienten oder stellte Kontakt zur zweitmeinungsgebenden Klinik her.

Die onlinebasierte Zweitmeinung fragten vor allem jüngere, männliche Patienten aus allen Bildungsschichten nach; 80 \% der Teilnehmer waren jünger als 70 Jahre, zwei Drittel waren Männer. Tatsächlich erkrankt mehr als die Hälfte der Betroffenen in 
- Tab. 1 Soziodemografische, tumor- und behandlungsspezifische Charakteristika der Studienteilnehmer insgesamt und differenziert nach Tumorlokalisation $(\mathrm{n}=95)$.

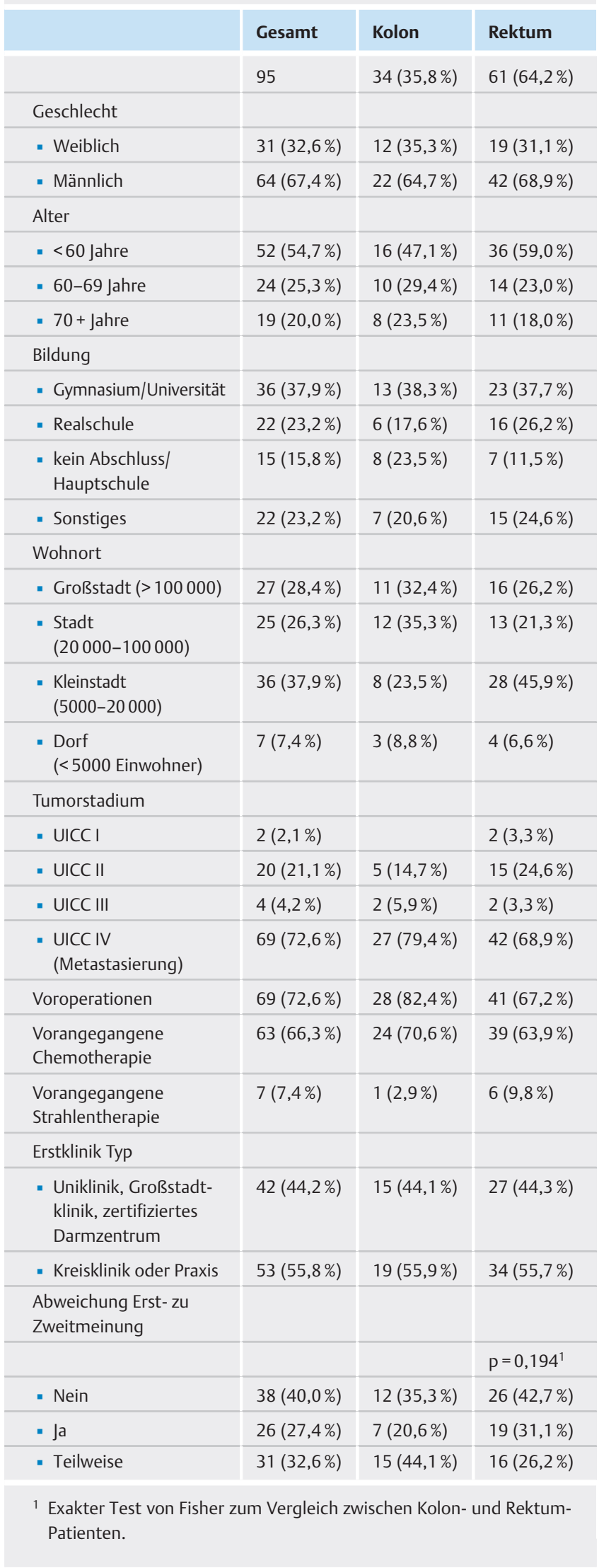

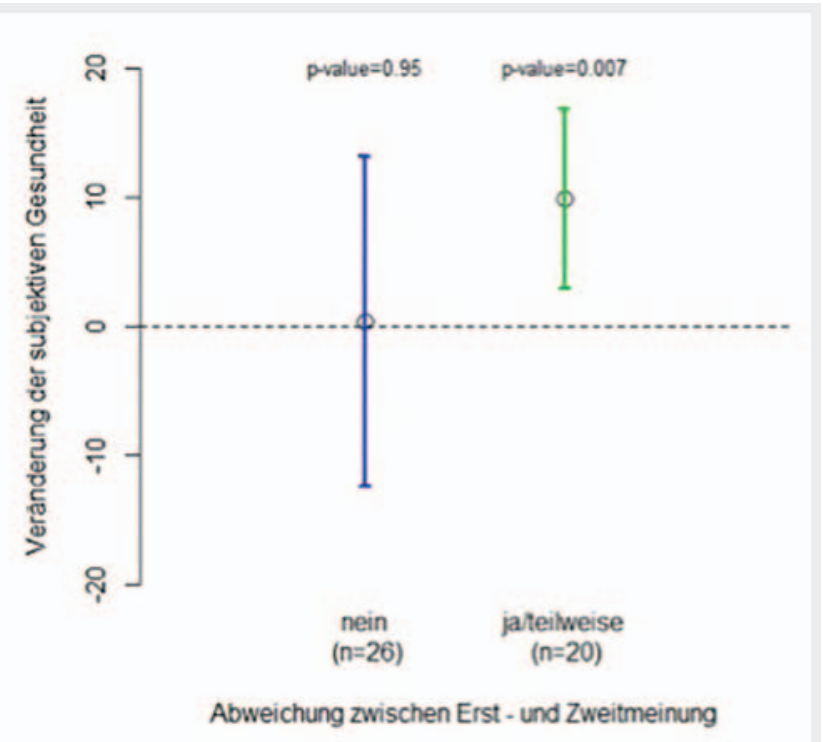

- Abb. 2 Mittlere Veränderung der subjektiven Gesundheit nach 3 Monaten in Abhängigkeit von der Abweichung der Therapieempfehlung zwischen Erstmeinung und Zweitmeinung (Flügel der ErrorPlots entsprechen dem Standardfehler SE, p-Werte resultieren aus den t-Tests für einfache Stichproben).

Deutschland nach dem 70. Lebensjahr, und über die Hälfte davon sind Frauen [1]. Allerdings nutzen jüngere Menschen häufiger eine onlinebasierte Gesundheitsberatung [15]. Über die Hälfte der teilnehmenden Patienten erhielt ihre initiale Therapieempfehlung nicht über die Vorstellung in einem Tumorboard, obwohl eine Vielzahl von Studien eine Verbesserung des onkologischen Outcomes durch die interdisziplinäre Fallbesprechung gezeigt hat [2-5]. Für Patienten ist es nach wie vor kaum möglich, sich selbstständig einem Tumorboard vorzustellen. Patienten, die mit ihrem behandelnden Arzt unzufrieden sind oder eine zweite Meinung zur Diagnose und Behandlung suchen, nutzen häufiger das Internet für gesundheitsspezifische Informationen [14]. Das Angebot einer qualitativen Zweitmeinung über ein Onlineportal könnte daher für Krebspatienten besonders hilfreich sein [16]. Der technologische und wissenschaftliche Fortschritt bedingt bei der multimodalen Behandlung der Patienten eine zunehmende Spezialisierung der beteiligten Disziplinen und setzt ein jeweiliges Expertenwissen voraus. Dies wirft die organisatorische Frage der ubiquitären Verfügbarkeit von Experten bei einer gewünschten heimatnahen Versorgung von Patienten auf. Das untersuchte Kollektiv gibt Hinweise auf einen möglicherweise großen Bedarf an qualifizierten Zweitmeinungen bei Patienten außerhalb der Ballungszentren, die ihren ersten Therapievorschlag von einer kleineren Klinik oder Praxis erhalten haben. Bei $60 \%$ der Patienten wich die Zweitmeinung teilweise oder vollständig von der Erstmeinung ab. Die Hälfte der betroffenen Patienten veränderte daraufhin ihr Behandlungsregime. Das Follow-up zeigte, dass sich bei diesen Patienten die subjektive Gesundheit bzw. die Lebensqualität signifikant verbesserte, und bestätigte insgesamt eine hohe Zufriedenheit mit der getroffenen Therapieentscheidung. Diese Ergebnisse unterstreichen den Bedarf und den Nutzen einer 
- Tab. 2 Therapieentscheidung, Zufriedenheit im 3-Monats-Follow-up und Entwicklung der subjektiven Gesundheit, insgesamt und differenziert nach Abweichung von Erst- und Zweitmeinung ( $n=57)$.

\begin{tabular}{|c|c|c|c|}
\hline & Gesamt & Abweichung keine & $\begin{array}{l}\text { Abweichung } \\
\text { ganz/teilweise }\end{array}$ \\
\hline & n (\%) & n (\%) & n (\%) \\
\hline & $57(60 \%)$ & $22(38,6 \%)$ & $35(61,4 \%)$ \\
\hline $\begin{array}{l}\text { Therapieentscheidung } \\
\text { (3-Monats-Follow-up) }\end{array}$ & & & $p=0,005^{1}$ \\
\hline - Therapiewechsel & $20(35,1 \%)$ & $3(13,6 \%)$ & $17(48,6 \%)$ \\
\hline - Therapie beibehalten & $30(52,6 \%)$ & $15(68,2 \%)$ & $15(42,8 \%)$ \\
\hline - Keine Therapie & $4(7 \%)$ & $1(4,5 \%)$ & $3(8,6 \%)$ \\
\hline - Keine Angabe & $3(5,3 \%)$ & $3(13,6 \%)$ & $0(0 \%)$ \\
\hline $\begin{array}{l}\text { Zufrieden mit der Entscheidung } \\
\text { (3-Monats-Follow-up) }\end{array}$ & & & $p=0,419^{1}$ \\
\hline - Ja & $52(91,2 \%)$ & $19(86,4 \%)$ & $33(94,3 \%)$ \\
\hline - Nein & $1(1,8 \%)$ & $1(4,5 \%)$ & $0(0,0 \%)$ \\
\hline \multirow[t]{3}{*}{ - Keine Angabe } & $4(7,0 \%)$ & $2(9,1 \%)$ & $2(5,7 \%)$ \\
\hline & Gesamt & Abweichung keine & $\begin{array}{l}\text { Abweichung } \\
\text { ganz/teilweise }\end{array}$ \\
\hline & Mittelwert (SD) & Mittelwert (SD) & Mittelwert (SD) \\
\hline Gesundheit Erstbefragung ( $\mathrm{n}=81$ ) & $51,9(22,1)$ & $45,4(22,0)$ & $55,8(21,4)$ \\
\hline Gesundheit 3-Monats-Follow-up $(n=51)$ & $60,3(22,6)$ & $50,0(20,2)$ & $68,1(21,4)$ \\
\hline $\begin{array}{l}\text { Gesundheit } 6 \text { Monate } \\
(n=29)\end{array}$ & $63,5(19,0)$ & $53,3(20,5)$ & $68,9(16,2)$ \\
\hline $\begin{array}{l}\text { Gesundheit } 12 \text { Monate } \\
(\mathrm{n}=28)\end{array}$ & $68,2(29,2)$ & $73,7(32,6)$ & $63,3(26,1)$ \\
\hline
\end{tabular}

- Tab. 3 Effekte der einflussreichen Charakteristika auf die Veränderung der subjektiven Gesundheit innerhalb von 3 Monaten (Multiple Regressionsanalyse, $n=46$ ).

\begin{tabular}{|l|l|l|}
\hline & Koeffizient (SE) & p-Wert \\
\hline Konstante & $24,20(9,22)$ & $0,012^{*}$ \\
\hline Gesundheit bei Baseline & $-0,65(0,13)$ & $<0,001^{*}$ \\
\hline $\begin{array}{l}\text { Abweichung zwischen } \\
\text { Erst- und Zweitmeinung } \\
\text { (deutlich oder teilweise) }\end{array}$ & $18,86(5,34)$ & $0,001^{*}$ \\
\hline Geschlecht (männlich) & $-11,70(5,32)$ & $0,034^{*}$ \\
\hline Alter & & \\
\hline - <50 Jahre & $17,47(8,51)$ & $0,047^{*}$ \\
\hline $.50-59$ Jahre & $16,23(7,31)$ & $0,032^{*}$ \\
\hline $.60-69$ jahre & $16,92(9,12)$ & 0,071 \\
\hline.$\geq 70$ Jahre & Referenz & - \\
\hline
\end{tabular}

Zweitmeinung und entsprechen damit anderen Untersuchungen zu Zweitmeinungsangeboten [17, 18].

Infolge der gesetzlichen Verankerung des Rechts auf eine Zweitmeinung sind vor allem in universitären Zentren die Zweitmeinungsanfragen gestiegen. Die Erstellung einer Expertenmeinung ist mit einem hohen personellen, logistischen und zeitlichen Aufwand verbunden, ohne entsprechende finanzielle Vergütung [16]. Hier hat sich in unserer Studie die Einbindung eines Case Managers als hilfreich erwiesen. Er garantierte, dass das die Zweitmeinung erstellende Expertengremium immer eine vollständige Akte vorgelegt bekam, und trug letztlich dazu bei, dass die Zweitmeinung bei 95 von 100 teilnehmenden Patienten innerhalb eines relativ kurzen Zeitraums erstellt werden konnte. Die teilnehmenden Kliniken sahen keine Schwierigkeiten darin, eine qualifizierte Zweitmeinung allein auf der Basis der strukturierten Elektronischen Patientenakte und ohne Gelegenheit, den Patienten persönlich in Augenschein zu nehmen, zu erstellen. Gleichzeitig ist die Akzeptanz des elektronischen Austausches von Gesundheitsdaten auch bei Patienten gestiegen [19].

SE: Standard-Fehler, $\mathrm{p}$-Wert des t-Tests, ${ }^{*} \mathrm{p}<0,05$ 


\section{Limitationen}

Die Pilotstudie wurde zwischen 2009 und 2011 primär initiiert, um eine Abschätzung der Machbarkeit und des Bedarfs einer onlinebasierten qualifizierten Zweitmeinung bei Patienten mit kolorektalem Karzinom zu erhalten. Die Fallzahl ergab sich damit aus der vorgegebenen Projektdauer. Aufgrund der aktuellen Diskussion zur Zweitmeinung bei Krebserkrankungen wurden die Daten aus dieser Pilotstudie nun auch ausführlicher hinsichtlich ihrer Ergebnisse bezüglich der Abweichung der Zweitmeinung und eines möglichen Einflusses auf die Lebensqualität statistisch analysiert. Limitierende Faktoren der Pilotstudie für diese statistischen Analysen sind sicherlich das Alter der Daten, die geringe Patientenzahl sowie das Fehlen einer Kontrollgruppe. Der hohe Anteil abweichender Zweitmeinungen und folgender Änderungen des Behandlungsregimes kann zum einen an einem hohen Anteil an Patienten liegen, die initial keinem interdisziplinären Tumorboard vorgestellt wurden, und zum anderen daran, dass vor allem unzufriedene Patienten häufiger das Internet für Gesundheitsfragen nutzen [14] und so auf diese Studie aufmerksam wurden. Dies war jedoch auch nicht der primäre Fokus der vorliegenden Studie.

\section{Fazit}

Das onlinebasierte Einholen einer qualifizierten Zweitmeinung zu einer komplexen Erkrankung wie Darmkrebs durch ein interdisziplinäres Tumorboard ist mithilfe einer strukturierten Elektronischen Patientenakte und mit einem Case Manager technisch und logistisch gut realisierbar. Dies könnte es in Zukunft ermöglichen, Patienten aller Alters- und Bildungsschichten flächendeckend zu erreichen. Die Erstellung einer zweiten Meinung durch ein interdisziplinäres Tumorboard hat einen positiven Einfluss auf die subjektive Gesundheit der Patienten und kann zu einer Verbesserung der Lebensqualität führen. Die Kombination aus onlinebasierter strukturierter EPA und zentraler Anlaufstelle Case Management führt sowohl bei Patienten als auch bei den in den Zweitmeinungskliniken behandelnden Ärzten zu großer Zufriedenheit hinsichtlich Logistik, Akzeptanz und Zeitraum bis zur Erstellung der Zweitmeinung.

\section{KERNAUSSAGEN}

- Die Erstellung einer qualifizierten Zweitmeinung zu einer komplexen Erkrankung wie Darmkrebs auf der Basis einer online übermittelten, strukturierten Elektronischen Patientenakte ist technisch und logistisch gut durchführbar.

- Ein Case Manager betreut und informiert die anfragenden Patienten und garantiert, dass das Tumorboard zur Erstellung der Zweitmeinung eine vollständige Akte vorgelegt bekommt.

- Erstmeinungen, die nicht von einem Tumorboard erstellt wurden, zeigen häufiger deutliche Abweichungen im Vergleich zur qualifizierten Zweitmeinung.

- Patienten, die ihre Therapie der Zweitmeinung anpassen, berichten eine signifikante Verbesserung der subjektiven Gesundheit.
- Die Kombination aus onlinebasierter strukturierter Elektronischer Patientenakte und zentraler Anlaufstelle Case Management führt sowohl bei Patienten als auch bei den Ärzten des Tumorboards zu großer Zufriedenheit.

Interessenkonflikt

Christa Maar ist geschäftsführender Vorstand der Felix Burda Stiftung. Udo E. Beckenbauer ist Vorstand der Firma HMO Health Management Online AG. Alle anderen Autoren geben an, dass kein Interessenskonflikt vorliegt.

Literatur

[1] Zentrum für Krebsregisterdaten im Robert Koch-Institut. Bericht zum Krebsgeschehen in Deutschland 2016. Berlin, 2016 http://www. krebsdaten.de/Krebs/DE/Content/Publikationen/Krebsgeschehen/ Krebsgeschehen_node.html (last accessed on 1 October 2019)

[2] Prades J, Remue E, van Hoof E et al. Is it worth reorganising cancer services on the basis of multidisciplinary teams (MDTs)? A systematic review of the objectives and organisation of MDTs and their impact on patient outcomes. Health Policy (Amsterdam, Netherlands) 2015; 119 (4): 464-474

[3] Junor E], Hole DJ, Gillis CR. Management of ovarian cancer: referral to a multidisciplinary team matters. Br J Cancer 1994; 70: 363-370

[4] Hong NJ, Wright FC, Gagliardi AR et al. Examining the potential relationship between multidisciplinary cancer care and patient survival: an international literature review. J Surg Oncol 2010; 102: 125-134

[5] Homayounfar K, Bleckmann A, Helms HJ et al. Discrepancies between medical oncologists and surgeons in assessment of resectability and indication for chemotherapy in patients with colorectal liver metastases. Br J Surg 2014; 101 (5): 550-557

[6] Ali J, Pieper D. Kaum aktuelle Daten zu Zweitmeinungsverfahren vorhanden - eine systematische Übersichtsarbeit. Gesundheitswesen 2017; 79 (10): 871-874

[7] Weyerstraß J, Prediger B, Neugebauer E et al. Erste Ergebnisse eines deutschen Zweitmeinungsportals zeigen neben einer hohen Patientenzufriedenheit große Diskrepanzen zwischen Erstempfehlung und Zweitmeinung. Z Evid Fortbild Qual Gesundhwes 2018; 133: 46-50

[8] Leitlinienprogramm Onkologie (Deutsche Krebsgesellschaft, Deutsche Krebshilfe, AWMF): S3-Leitlinie Kolorektales Karzinom, Langversion 1.0, AWMF Registernummer: 021-007OL. http://leitlinienprogrammonkologie.de/Leitlinien.7.0.html

[9] UICC (International Union Against Cancer). TNM Classification of malignant tumours. Sobin LH, Wittekind Ch, (eds) 6th ed New York: Wiley; 2002

[10] Aaronson NK, Ahmedzai S, Bergman B et al. The European Organization for Research and Treatment of Cancer QLQ-C30: A quality-of-life instrument for use in international clinical trials in oncology. J Natl Cancer Inst 1993; 85: 365-376

[11] McLachlan SA, Devins GM, Goodwin PJ. Validation of the European Organization for Research and Treatment of Cancer Quality of Life Questionnaire (QLQ-C30) as a measure of psychosocial function in breast cancer patients. European journal of cancer (Oxford, England: 1990) 1998; 34 (4): 510-517

[12] Renahy E, Parizot I, Chauvin P. Determinants of the frequency of online health information seeking: results of a web-based survey conducted in France in 2007. Inform Health Soc Care 2010; 35 (1): 25-39 
[13] Heiman H, Keinki C, Huebner J. EHealth literacy in patients with cancer and their usage of web-based information. J Cancer Res Clin Oncol 2018; 144 (9): 1843-1850

[14] Tustin N. The role of patient satisfaction in online health information seeking. J Health Commun 2010; 15 (1): 3-17

[15] Baumann E, Czerwinski F, Reifegerste D. Gender-specific determinants and patterns of online health information seeking: Results from a representative German health survey. J Med Internet Res 2017; 19 (4): e92

[16] Homayounfar K, Lordick F, Ghadimi M. Qualitätssicherung: Multidisziplinäre Tumorboards - trotz Problemen unverzichtbar. Dtsch Arztebl 2014; 111 (22): A-998 / B-852 / C-806
[17] Hillen MA, Medendorp NM, Daams JG et al. Patient-driven second opinions in oncology: A systematic review. . Oncologist 2017; 22 (10): 11971211

[18] Clauson J, Hsieh YC, Acharya S et al. Results of the Lynn Sage SecondOpinion Program for local therapy in patients with breast carcinoma. Changes in management and determinants of where care is delivered. Cancer 2002; 94 (4): 889-894

[19] Kim KK, Joseph JG, Ohno-Machado L. Comparison of consumers' views on electronic data sharing for healthcare and research. J Am Med Inform Assoc 2015; 22 (4): 821-830 